\title{
Neonatal Rat; A Suitable Animal Model for Experimental Cryptosporidiosis
}

\author{
Seyede Manizhe Heidarnejadi, ${ }^{1,2}$ Abdollah Rafiei, ${ }^{1,2,}{ }^{*}$ Manoochehr Makvandi, ${ }^{2,3}$ Majid Pirestani, ${ }^{4}$ Jasem \\ Saki, ${ }^{1}$ and Ataallah Ghadiri ${ }^{5}$ \\ ${ }^{1}$ Infectious and Tropical Diseases Research Center, Health Research Institute, Ahvaz Jundishapur University of Medical Sciences, Ahvaz, IR Iran \\ ${ }^{2}$ Department of Medical Parasitology, School of Medicine, Ahvaz Jundishapur University of Medical Sciences, Ahvaz, IR Iran \\ ${ }^{3}$ Department of Virology, School of Medicine, Ahvaz Jundishapur University of Medical Sciences, Ahvaz, IR Iran \\ ${ }^{4}$ Department of Parasitology and Entomology, Faculty of Medical Sciences, Tarbiat Modares University, Tehran, IR Iran \\ ${ }^{5}$ Department of Immunology, School of Medicine, Ahvaz Jundishapur University of Medical Sciences, Ahvaz, IR Iran \\ "Corresponding author: Abdollah Rafiei, Department of Medical Parasitology, Infectious and Tropical Diseases Research Center, Health Research Institute, Ahvaz Jundishapur \\ University of Medical Sciences, Zip Code: 61357-15794, Ahvaz, IR Iran. Tel: +98-6113337077, Fax: +98-6113367868, E-mail: rafieiabdollah@yahoo.com
}

Received 2017 February 07; Revised 2017 March 17; Accepted 2017 April 12.

\begin{abstract}
Background: Cryptosporidiosis is a major public health problem for neonatal livestock worldwide. Cryptosporidium parvum infects intestinal epithelial cells via contaminated food or drinking water and leads to cryptosporidiosis. Most of the animal model studies on infectivity of $C$. parvumare conducted on the neonatal mice.

Objectives: The current study aimed at evaluating the infectivity of $C$. parvum in neonatal rat as an animal model.

Methods: A dose of 100,000 to 120,000 C. parvum oocysts (Iowa strain, BTF Company, Sydney, Australia) was orally inoculated in a group of 30 neonatal Wistar rats aged 2 days old. Eight days postinfection, jejunum, ileum, cecum, colon, and rectum were removed and contents were homogenized and purified using sucrose gradient method.

Results: Our results indicated that 6 to 12 million C. parvum was found per rat

Conclusions: Analysis of the study results revealed that the neonatal rat could be used as an alternative animal model to investigate C. parvum.
\end{abstract}

Keywords: Iowa Strain, Purification, Neonatal Rat, Cryptosporidium parvum

\section{Background}

Cryptosporidiosis is a major public health problem in immunosuppressed human being as well as in neonatal livestock worldwide. Cryptosporidium parvum is transmitted via contaminated food or water, which may infect intestinal epithelial cells and results in cryptosporidiosis. Cryptosporidiosis is recognized as an emerging infectious disease by the centers for disease control and prevention (1). It is a self-limited disease, but may lead to a chronic infection and even become life-threatening in the immunocompromised individuals. It is estimated that cryptosporidiosis causes 5 to 10 million deaths each year (2); $13 \%$ to $74 \%$ prevalence is reported in HIV-positive children with diarrhea in sub-Saharan Africa (3), and 7. $8 \%$ to $10.3 \%$ in patients with HIV aged $>85$ years in the United States (4).

Since the number of patients with AIDS is increasing in the developing countries, the significance of prevention and treatment of C. parvum is remarkable. Cryptosporidium parvum is a waterborne and zoonotic infection that can be transmitted to human via infected cattle, calves, sheep, etc. (5-8). In the developing countries, a major ob- stacle for disease control is the lack of effective methods to curb cryptosporidiosis and reduce environmental contamination with oocysts (9). Cryptosporidium parvum can be spread via food handlers, contaminated foods, fruits, vegetables, and unpasteurized cow milk (10-13). The epidemiology of C. parvum is investigated in Iran. The detection of C. parvum reported in cows is about $70.8 \%$ (14). The incidence of C. parvum in domestic animals was reported 59\%; also the incidence of cryptosporidiosis in the exposed children was estimated $14.5 \%$ in the Southwest of Iran $(14,15)$. In the light of related diseases to C. parvum, it is required to conduct the periodical studies on the farm animals to prevent the spread of C. parvum in humans and animals.

Several animal model systems are described for clinical manifestation and drug designing against $C$. parvum so for, animals models included newborn BALB/c mice (16), rat, guinea pigs (17), calves (18), hamsters (19), and Arc/Swiss mice (20). At present, rats are used as animal model laboratory for in vivo studies of C. parvum to determine the incubation period, pathogenesis, immune response, and drug testing. Therefore, the current study aimed at evaluating the infectivity of C. parvum in the rats as an animal model. 


\section{Objectives}

The current study aimed at evaluating the infectivity of C. parvum in the rats as an animal model.

\section{Methods}

Viable C. parvum oocyst Iowa strain was purchased from BTF Company (Sydney, Australia). Thirty neonatal Wistar rats aged 2 days were purchased from Razi vaccine and serum research institute, Karaj, Iran. Adose of 100,000 - 1,200,000 C. parvum was orally inoculated using gastric tube. Eight days post infection, the neonatal rats were scarified with ether and jejunum, ileum, caecum, colon, and rectum were removed; the contents were pooled and put in a Petri dish. Contents were homogenized (with an UltraTurrax homogenizer, 3 times for 10 second), Sputasol (2 $\mathrm{mL} / 50 \mathrm{~mL}$ phosphate-buffered saline for 10 to 13 rats) was added and homogenized vigorously using bar mix (21).

The contents were mixed with $0.2 \mathrm{~mL}$ of homogenized suspension and stained by the Ziehl-Neelsen technique. The final purification of oocysts was conducted by sucrose gradients described by Lumb et al. Briefly, the Sheather sucrose solution was diluted 1:2 and 1:4 with sterile PBS (0.1 $\mathrm{M}, \mathrm{pH}$ 7.2). The sucrose gradient was prepared by placing $10 \mathrm{~mL}$ of the $1: 2$ solution into $50 \mathrm{~mL}$ of polypropylene centrifuge tubes (Falcon; Becton Dickinson, Franklin Lakes, NJ) and, then, slowly adding $10 \mathrm{~mL}$ of the $1: 4$ solution. A $30 \mathrm{~mL}$ of the oocyst suspension $\left(1.5 \times 10^{6} / \mathrm{mL}\right)$ was layered onto the $1: 4$ gradient. The sucrose gradient was centrifuged at $1,700 \mathrm{X}$ for 30 minutes at $48^{\circ} \mathrm{C}$. Following the centrifugation, the potassium dichromate layer and half of the 1:4 gradient were collected with a pipette and, then, discarded. The remainder of the $1: 4$ gradient and $0.5 \mathrm{~mL}$ of the 1:2 gradient above the interface were recovered and dispersed into $80 \mathrm{~mL}$ of PBS, and the purified oocysts were counted. The suspension of oocysts per $1 \mathrm{~mL}$ was enumerated by a Neubauer hemocytometer.

\section{Results and Discussion}

All inoculated neonatal rats showed signs such as watery stool, diarrhea, dehydration, weight loss, with a relative reduction in appetite. The shedding of oocyst in the feces of neonatal rat was observed at 8 days postinfection and confirmed by the Ziehl-Neelsen staining method (Figure 1). The results of oocysts purification by sucrose gradient revealed that about 6 to 12 million oocysts were observed in the samples, 8 days after infection.

The presence of an alternative animal model is important to evaluate further investigations on pathogenesis, immunological, drugs, and vaccine aspects. In the present

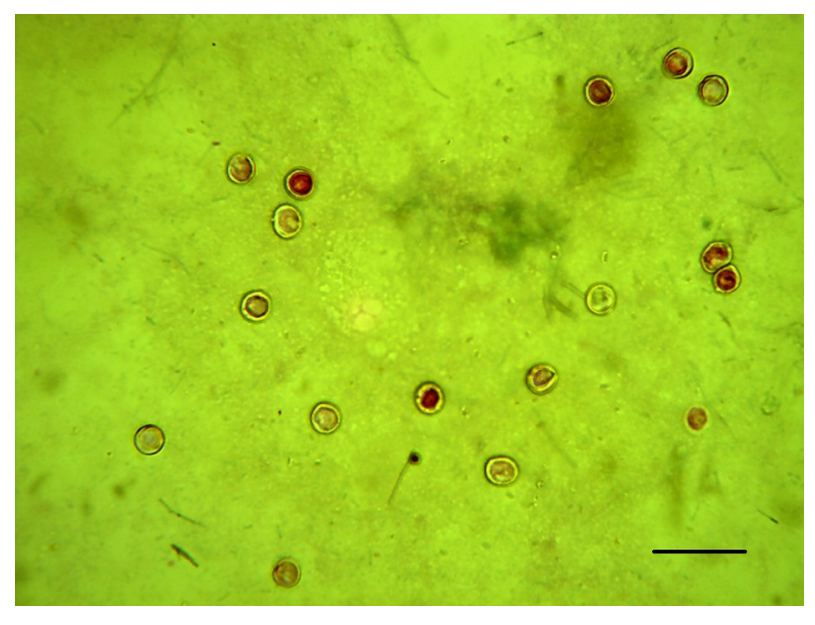

Figure 1. Ziehl-Neelsen Staining, Cryptosporidium parvum Oocysts in the Intestine Material of the Infected Neonatal Wistar Rats (Scale Bars $20 \mu$ )

study the number of oocysts in the neonatal rat was 60 to 100 times higher than the orally inoculated dose. The outcomes of the current study help to maintain infectivity of the oocytes as well as increasing and restoring the number of oocysts at $4^{\circ} \mathrm{C}$ for future studies. To purify the oocysts from the stool of postinfected rats, each step was carefully evaluated and recorded (22); mass production of $C$. parvum oocyst from postinfected mice as an animal model and its storage and maintenance at $4^{\circ} \mathrm{C}$ for a limited time were reported. Most of the studies indicated that mice are suitable as an animal model for infectivity, pathogenesis, and other investigations of the C. parvum oocysts $(20,23)$.

According to the results of the current study, clinical signs and symptoms of diarrheal were observed in the neonatal rats 8 days after the oral inoculation of C. parvum oocysts. In addition, the observation of 6 to 12 million oocysts indicated the dramatically increase of oocytes 60 to 100 times more than the initial does in the stools of postinfected neonatal mice. Based on the current study results, neonatal rat could be also used as an animal experimental model for the investigation of C. parvum in vivo. Mirza Qhavi et al. reported the peak of infection intensity of C. parvum oocysts with similar patterns of symptoms in neonatal mice and rats on the day 9 . They concluded that it was easier to handle rats than mice during the procedure and rats were preferable for in vivo study to mice (24). Petry et al. observed high intensive infectivity of $10^{9} \mathrm{C}$. parvum oocyst in the stools of a group of 10 mice 8-10 days old immunosuppressed with dexamethasone (23). Current and Reese inoculated 3 isolates of C. parvum oocysts obtained from the cave of an immunocompetent individual, and a patient with HIV in mice and reported no difference on the 
clinical signs and symptoms among the mice infected with C. parvum oocysts with different origins (25).

The current study purified the C. parvum oocysts from the homogenized intestine of neonatal rats using sucrose gradient and recovered 6 to 12 million oocysts. Meloni and Thompson et al. purified 6 to 15 million C. parvum oocysts from the stool of infected neonatal Arc/Swiss mice using Ficoll gradient (20), which was in agreement with the current study findings. Emilio E et al. purified C. parvum oocysts from stool samples of a newborn goat infected with C. parvum using 6\%, 16\%, and $28 \%$ (w/v) $\mathrm{KBr}$ gradient in Tris-ethylenediaminetetraacetic acid (EDTA) buffer (26).

\section{Conclusions}

The results of the current study showed that 6 to 12 million oocysts were observed in the stool of 2-day days neonatal rats after infection with C. parvum (60 to 100 times more than the initial does). Thus, the 2-day old neonatal rats could be also used as an experimental animal model for $C$. parvum investigations.

\section{Acknowledgments}

The current manuscript is part of a research work, grant no. 94147, conducted by Seyede Manizhe Heidarnejadi as her PhD thesis in parasitology supported by the infectious diseases research center, the health research institute of Ahvaz Jundishapur University of Medical Sciences, Ahvaz, Iran. Authors would like to thank Dr. Ali Zarei Mahmoudabadi, department of medical mycology, school of medicine, Ahvaz Jundishapur University of Medical Sciences, and Dr. Amir Abdoli, departments of parasitology and entomology, Tarbiat Modares University, Tehran, Iran, for their valuable suggestions and comments on the manuscript.

\section{References}

1. Bryan RT, Pinner RW, Gaynes RP, Peters CJ, Aguilar JR, Berkelman RL. Addressing Emerging Infectious Disease Threats: A Prevention Strategy for the United States: Executive Summary. Morbid Mortal Week Rep Recommend Rep. 1994:i-18.

2. Nemes Z. [Diarrhea from the infectologist's point of view]. Orv Hetil. 2009;150(8):353-61. doi: 10.1556/OH.2009.28549. [PubMed: 19218145].

3. Mor SM, Tzipori S. Cryptosporidiosis in children in Sub-Saharan Africa: a lingering challenge. Clin Infect Dis. 2008;47(7):915-21. doi: 10.1086/591539. [PubMed: 18715159].

4. Mor S M, DeMaria A, Griffiths J K, Naumova E N. Cryptosporidiosis in the Elderly Population of the United States. Clin Infect Dis. 2009;48(6):698-705. doi:10.1086/597033.

5. Bowman DD, Lucio-Forster A. Cryptosporidiosis and giardiasis in dogs and cats: veterinary and public health importance. Exp Parasitol. 2010;124(1):121-7. doi: 10.1016/j.exppara.2009.01.003. [PubMed: 19545532].
6. Xiao L. Molecular epidemiology of cryptosporidiosis: an update. Exp Parasitol. 2010;124(1):80-9. doi: 10.1016/j.exppara.2009.03.018. [PubMed: 19358845].

7. Xiao L, Ryan UM. Cryptosporidiosis: an update in molecular epidemiology. Curr Opin Infect Dis. 2004;17(5):483-90. [PubMed:15353969].

8. Chalmers RM, Smith RP, Hadfield SJ, Elwin K, Giles M. Zoonotic linkage and variation in Cryptosporidium parvum from patients in the United Kingdom. Parasitol Res. 2011;108(5):1321-5. doi:10.1007/s00436010-2199-x. [PubMed: 21193928].

9. Carey CM, Lee H, Trevors JT. Biology, persistence and detection of Cryptosporidium parvum and Cryptosporidium hominis oocyst. Water Res. 2004;38(4):818-62. doi:10.1016/j.watres.2003.10.012. [PubMed: 14769405].

10. Robertson LJ, Gjerde B. Occurrence of parasites on fruits and vegetables in Norway. J Food Prot. 2001;64(11):1793-8. [PubMed: 11726161].

11. Robertson LJ, Chalmers RM. Foodborne cryptosporidiosis: is there really more in Nordic countries?. Trends Parasitol. 2013;29(1):3-9. doi: 10.1016/j.pt.2012.10.003. [PubMed: 23146217].

12. Amoros I, Alonso JL, Cuesta G. Cryptosporidium oocysts and giardia cysts on salad products irrigated with contaminated water.J Food Prot. 2010;73(6):1138-40. [PubMed: 20537274].

13. Harper CM, Cowell NA, Adams BC, Langley AJ, Wohlsen TD. Outbreak of Cryptosporidium linked to drinking unpasteurised milk. Commun Dis Intell Q Rep. 2002;26(3):449-50. [PubMed:12416712].

14. Pirestani M, Sadraei J, Dalimi Asl A, Zavvar M, Vaeznia H. Molecular characterization of Cryptosporidium isolates from human and bovine using 18s rRNA gene in Shahriar county of Tehran, Iran. Parasitol Res. 2008;103(2):467-72. doi: 10.1007/s00436-008-1008-2. [PubMed: 18478266].

15. Heidarnegadi S, Mohebali M, Maraghi S, Babaei Z, Farnia S, Bairami A, et al. Cryptosporidium spp. Infection in human and domestic animals. Iran J Parasitol. 2012;7(1):53-8. [PubMed: 23133472].

16. Ahmadian N, Pashaei-Asl R, Ahmadian M, Rahmati-Yamchi M, Shahabi S, Vazini H. Immune response of newborn BALB/C mice to Cryptosporidium infection. J Parasit Dis. 2016;40(3):1066-70. doi: 10.1007/s12639-014-0635-z. [PubMed: 27605838].

17. Chrisp CE, Reid WC, Rush HG, Suckow MA, Bush A, Thomann MJ. Cryptosporidiosis in guinea pigs: an animal model. Infect Immun. 1990;58(3):674-9. [PubMed: 2307517].

18. O'Brien CN, Jenkins MC. A rapid method for producing highly purified Cryptosporidium parvum oocysts. J Parasitol. 2007;93(2):434-6. doi: 10.1645/GE-1020R.1. [PubMed: 17539434].

19. Kim CW. Laboratory animal models for experimental cryptosporidiosis: a minireview. Res Rev Parasitol. 1994;54(1):13-28.

20. Meloni BP, Thompson RC. Simplified methods for obtaining purified oocysts from mice and for growing Cryptosporidium parvum in vitro. J Parasitol. 1996;82(5):757-62. [PubMed: 8885885].

21. Current WL. In: Parasitici nfections in the compromised host. Walzer RMG, editor. New York: Marcel Dekker; 1989. pp. 281341.Cryptosporidiumsp.

22. Sherwood D, Angus KW, Snodgrass DR, Tzipori S. Experimental cryptosporidiosis in laboratory mice. Infect Immun. 1982;38(2):471-5. [PubMed: 7141705].

23. Petry F, Robinson HA, McDonald V. Murine infection model for maintenance and amplification of Cryptosporidium parvum oocysts. JClin Microbiol. 1995;33(7):1922-4. [PubMed: 7665672].

24. Mirza-Qavami SM, Sadraei J.. An examination of Cryptosporidium parvum infection in neonate BALB/C mice and rats. Jundishapur J Microbiol. 2011;4(3):185-90.

25. Current WL, Reese NC. A comparison of endogenous development of three isolates of Cryptosporidium in suckling mice. J Protozool. 1986;33(1):98-108. [PubMed: 3959014].

26. Entrala E, Molina-Molina JM, Rosales-Lombardo MJ, Sánchez-Moreno M, Mascaró-Lazcano C. Cryptosporidium parvum: oocysts purification using potassium bromide discontinuous gradient. Vet Parasitol. 2000;92(3):223-6. doi: 10.1016/s0304-4017(00)00315-0. 